\title{
Reconhecimento de Dígitos em Imagens de Medidores de Energia no Contexto de um Aplicativo de Autoleitura
}

\author{
Arthur Costa Serra* João Vitor F. França* \\ Ricardo C. S. Marques* Weslley Kelson R. Figueredo* \\ Artur Bernardo Silva Reis* Italo Francyles S. da Silva* \\ Simara Vieira da Rocha* Aristófanes Correa Silva* \\ Eliana Márcia G. Monteiro** Italo Fernandes S. da Silva ** \\ Márcia Izabel A. da Silva ${ }^{* *}$ Jose Messias dos Santos** \\ * Núcleo de Computação Aplicada \\ Universidade Federal do Maranhão (UFMA) - São Luís, MA, Brasil \\ (e-mail: \{arthursrr, jvitorfranca, ricardo.marques, weslley.kelson, \\ arturberna, francyles, simara.rocha, ari\}@nca.ufma.br) \\ ** Equatorial Energia - São Luís, MA, Brasil \\ (e-mail: \{ eliana.monteiro, italo.silva, \\ marcia.silva,jose.messias\}@cemar-ma.com.br)
}

\begin{abstract}
According to Brazilian Electricity Regulatory Agency (ANEEL), non-technical losses are those related to energy theft and access impediment to consumer units. A feasible and low cost alternative for reducing these failures would be the reading done by customers which is called self-reading. This process includes the use of digital platforms and the customer would register and send consumption information. A primordial step of this process is the digits automatic recognition in energy meters through images. This work proposes a computational method to perform this task. Histogram of Oriented Gradients (HoG) and Local Self-similarity (LSS) descriptors are combined and used with Support Vector Machine (SVM) classifier. The proposed method obtains accuracy of $97.90 \%$ and $96.72 \%$ respectively for digits recognition in digital and analogical energy meters.

Resumo: Segundo a Agência Nacional de Energia Elétrica (ANEEL), perdas não-técnicas são aquelas relacionadas a furtos de energia e impedimento de acesso às unidades consumidoras. Uma alternativa viável e de menor custo para a redução dessas falhas seria a leitura realizada pelo próprio consumidor, denominada de autoleitura. Esse processo engloba o uso de plataformas digitais, por meio das quais o consumidor registraria e enviaria as informações de consumo. Uma etapa primordial desse processo é o reconhecimento automático de dígitos em medidores por meio de imagens. Este trabalho propõe um método computacional para a realização dessa tarefa. São utilizados os descritores de característica Histogram of Oriented Gradients(HoG) e Local Self-similarity(LSS) de forma combinada e o classificador Máquina de Vetores de Suporte (SVM). O método alcança acurácia de $97,90 \%$ e $96,72 \%$, respectivamente, para o reconhecimento de dígitos em medidores digitais e analógicos.
\end{abstract}

Keywords: Energy Consumption, Self-reading, Recognition, Image Processing, Machine Learning, HoG, LSS, SVM

Palavras-chaves: Consumo de Energia, Autoleitura, Reconhecimento, Processamento de imagens, Aprendizado de Máquina, HoG, LSS, SVM

\section{INTRODUÇÃO}

No Brasil, o consumo de energia elétrica tem crescido bastante nos últimos anos. De acordo com estudos realizados pela Empresa de Pesquisa Energética (EPE), o consumo nacional de energia elétrica atingiu, em 2018, cerca de

\footnotetext{
^ Este trabalho contou com o apoio do projeto Autoleitura, financiado pelo grupo Equatorial Energia, no âmbito do Programa de P \& D da Agência Nacional de Energia Elétrica (ANEEL) N ${ }^{\circ}$ PD-00037$0032 / 2018$.
}

472.200 GWh (gigawatts). O consumo residencial corresponde a $28 \%$ deste valor (ANEEL, 2019). Isso é justificado, por exemplo, pelo aumento no número de domicílios que passará de 75 milhões em 2020 segundo previsões (Vidinich and Nery, 2009).

A Agência Nacional de Energia Elétrica (ANEEL) aponta que podem ocorrer perdas técnicas e não-técnicas no processo de distribuição de energia (ANEEL, 2019). As perdas técnicas relacionam-se à quantidade de energia elétrica dissipada entre os suprimentos de energia da distribui- 
dora e os pontos de entrega (unidades consumidoras ou distribuidoras supridas). As perdas não associadas com as situações descritas, anteriormente, são chamadas de nãotécnicas. Furtos de energia, impedimento de acesso às unidades consumidoras e erros de medição e de processamento de faturamento são exemplos desse tipo de perda.

Em geral, as perdas não-técnicas são descobertas durante o processos de leitura e fiscalização do consumo de energia. A Companhia Energética do Maranhão (CEMAR) e as Centrais Elétricas do Pará S.A (CELPA) utilizam um dispositivo móvel e uma impressora portátil para coletar as informações de consumo e imprimir a fatura dos clientes. Para isso, o leiturista precisa informar manualmente o consumo tal qual é mostrado no registro de energia, o que faz esse processo suscetível a erros, gerando, portanto, inconsistências no faturamento mensal.

Uma alternativa para evitar erros seria a utilização de medidores inteligentes, mas este equipamento tem um custo elevado e a troca de todo o parque de equipamentos de medição (incluindo medidores analógicos e digitais) por esta tecnologia seria uma solução a longo prazo. Outra alternativa viável e de menor custo, seria a leitura realizada pelo próprio consumidor, denominada de autoleitura. Este processo engloba o uso de plataformas digitais, como sites ou aplicativo desenvolvido para dispositivo móvel, por onde o consumidor registraria e enviaria as informações de consumo.

Existem várias vantagens no processo de autoleitura. Pode-se destacar um estreitamento na relação de confiança entre companhias e consumidores, bem como a diminuição dos custos das companhias e a redução dos erros de leituras, principalmente em áreas rurais ou de difícil acesso.

A autoleitura também promove ao consumidor um maior controle sobre o seu consumo de energia. Uma vez obtido o valor do consumo, a companhia pode emitir a fatura ou fazer uma simulação da mesma, garantindo agilidade no processo de pagamento da fatura.

Nesse cenário, métodos computacionais capazes de realizar, automaticamente, a leitura do consumo de energia das unidades consumidoras por meio de análise de imagem podem auxiliar na aferição do consumo, facilitando e agilizando esse processo, tornando-o menos passível de erros.

Um método computacional de autoleitura por meio de imagem se dá, primeiramente, com a captura da fotografia do display do medidor. Em seguida, cada dígito é segmentado e, por fim, identificados para fins de geração de fatura. Baseando-se nessa proposta, este trabalho apresenta, portanto, um método para o reconhecimento de dígitos em imagens dos medidores de energia utilizados pelas companhias CEMAR e CELPA, visando contribuir com mais eficiência ao processo de leitura e também com a diminuição de perdas relacionadas ao faturamento.

O método utiliza técnicas de processamento de imagem e inteligência computacional para a extração de características e o reconhecimento dos dígitos tanto em medidores analógicos quanto em digitais, sendo parte integrante de uma aplicação, em desenvolvimento, para a implantação do processo de autoleitura.
Este trabalho está estruturado da seguinte forma: a Seção 2 apresenta trabalhos relacionados a este; a Seção 3 mostra o conjunto de imagens utilizado; a Seção 4 explica o método proposto; a Seção 5 discute os resultados obtidos por este estudo; e, a Seção 6 apresenta as conclusões.

\section{TRABALHOS RELACIONADOS}

$\mathrm{Na}$ literatura, existem alguns trabalhos com propostas para a realização automática da leitura do consumo de unidades consumidoras de energia elétrica baseado em imagens. Parthiban and Palanisamy (2013) utilizam cada pixel da imagem do dígito como entrada para uma rede neural artificial responsável pelo reconhecimento. Zhang et al. (2016) apresenta uma proposta de localização e segmentação dos dígitos utilizando detecção de borda, componentes conectados, e morfologia matemática.

Quintanilha et al. (2017) propõem o reconhecimento baseado em características extraídas com Histogram of Oriented Gradients (HoG) em conjunto com o classificador Máquina de Vetores de Suporte (SVM). Os autores realizam experimentos com medidores analógicos obtendo acurácia de $79,52 \%$.

Em nosso trabalho, propomos uma evolução do trabalho de Quintanilha et al. (2017), pois, abordamos o reconhecimento de dígitos não somente em medidores analógicos, como também em medidores digitais, utilizando a combinação de características extraídas via HoG e Local SelfSimilarity (LSS).

\section{BASE DE IMAGENS}

As companhias CEMAR e CELPA utilizam medidores analógicos e digitais para aferir o consumo de energia dos clientes. Exemplos desses medidores podem ser visualizados na Figura 1. No geral, esses dispositivos encontramse dentro de uma caixa protetora com uma parte transparente para que o leiturista possa conferir os dígitos no display e realizar a leitura de consumo.

As imagens de medidores foram adquiridas sob condições irrestritas de iluminação e orientação, simulando o processo de autoleitura. É importante ressaltar que, eventualmente, a caixa protetora do medidor pode conter avarias pela ação de agentes externos. Isso pode dificultar a visualização dos dígitos. Não obstante, imagens com essas características não foram descartadas.

A base de dados utilizada neste trabalho contém imagens dos dígitos de 0 a 9 , separados entre os tipos digital e analógico. Os dígitos foram recortados manualmente das imagens de displays, cuja segmentação também se deu de forma manual a partir das imagens dos medidores. A Figura 2 mostra exemplos de dígitos de medidores digitais. Este conjunto contém 10.613 imagens.

A Figura 3 apresenta exemplos de dígitos observados em medidores analógicos. A CEMAR e a CELPA utilizam 7 modelos diferentes de medidores analógicos, e isso garante bastante variabilidade para a base de imagens. Os dígitos de cada modelo de medidor têm características diferentes entre si. Sendo assim, a base de dígitos analógicos foi organizada, primeiramente, baseada nas fontes dos dígitos e, 

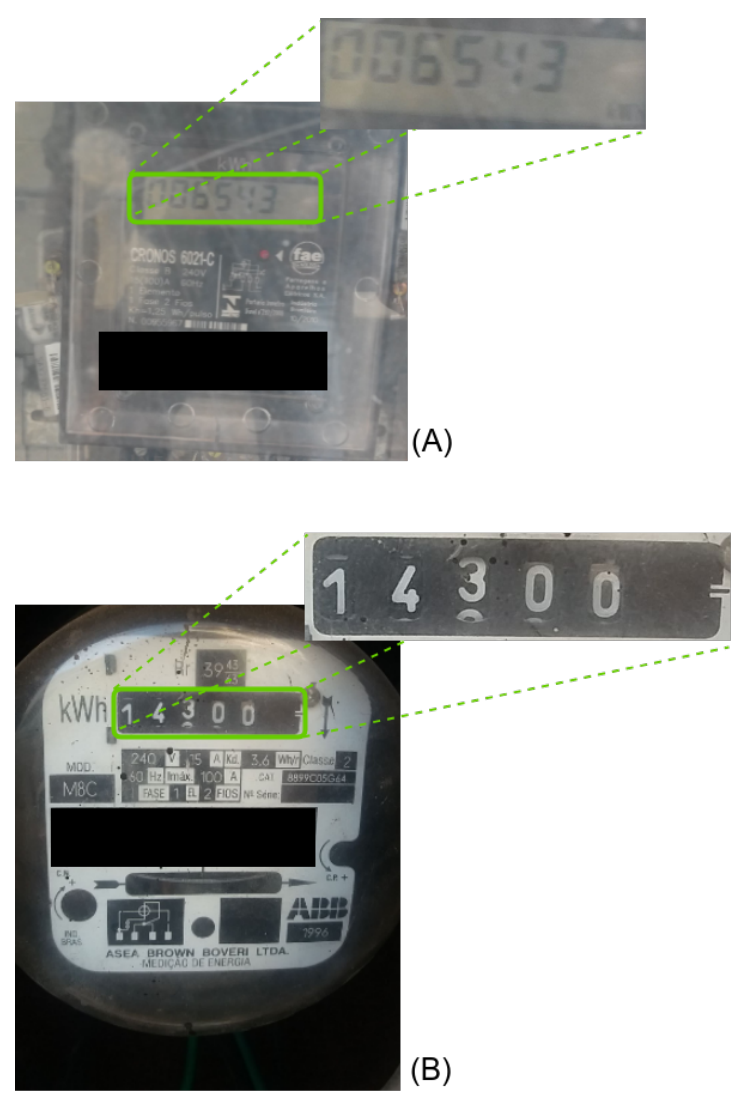

Figura 1. Exemplos de modelos de medidores digital (A) e analógico (B) utilizados pelas companhias.

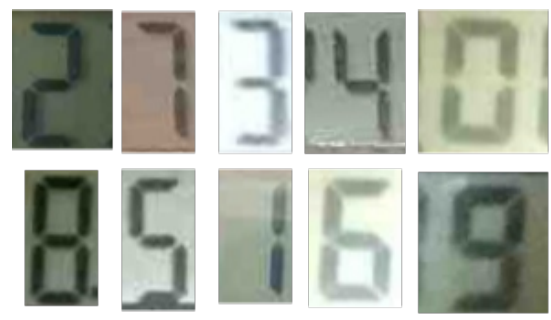

Figura 2. Exemplos de dígitos oriúndos de medidores digitais.

em seguida, pelas classes de números. Este conjunto possui 18.139 imagens divididos de acordo como apresentado pela Tabela 1.

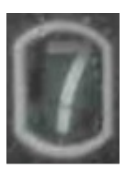

C1

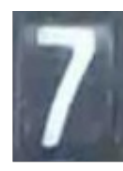

C2

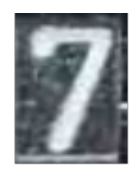

C3

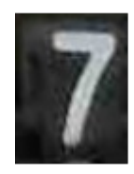

C4

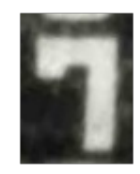

C5

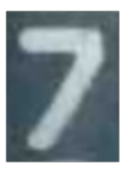

C6

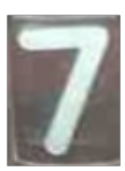

C7
Figura 3. Exemplos de dígitos oriúndos de medidores analógicos das classe C1 a C7.
Tabela 1. Quantidade de dígitos analógicos por tipo de medidor.

\begin{tabular}{cc}
\hline Medidores & Total \\
\hline Classe 1 & 2410 \\
Classe 2 & 3149 \\
Classe 3 & 4455 \\
Classe 4 & 3804 \\
Classe 5 & 622 \\
Classe 6 & 2955 \\
Classe 7 & 744 \\
\hline
\end{tabular}

\section{MÉTODO PROPOSTO}

Este trabalho tem como objetivo o reconhecimento de dígitos em medidores digitais e analógicos. Assim sendo, a proposta apresentada insere-se em um pipeline como um passo posterior às etapas de detecção do display e de segmentação dos dígitos.

O método proposto para o reconhecimento de dígitos é composto pela sequência de etapas ilustradas pela Figura 4 .

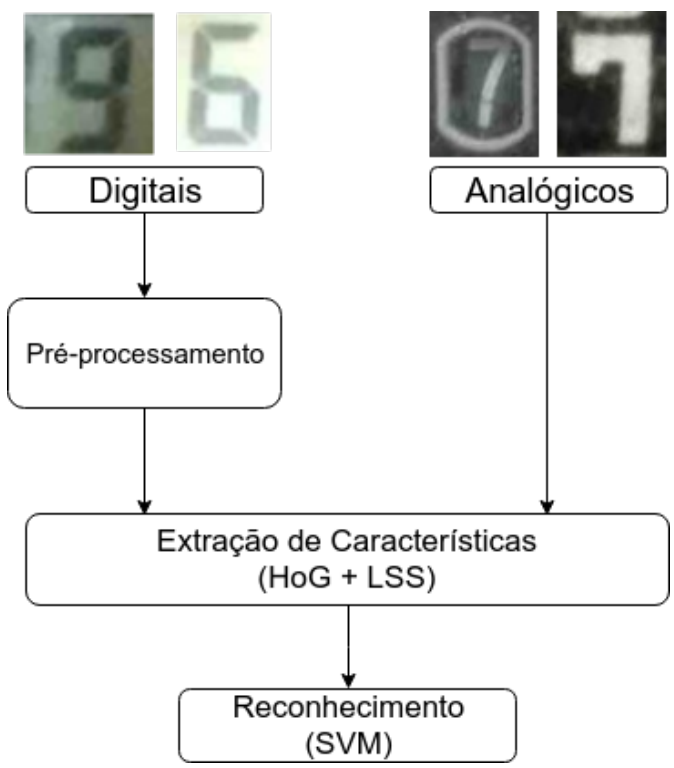

Figura 4. Etapas do Método Proposto

As imagens dos dígitos previamente segmentadas são a entrada para o método. Os dígitos provenientes de medidores digitais passam por um pré-processamento. Aqueles oriundos de medidores analógicos, não. Em seguida, as imagens são passadas para a etapa de extração de característica e estas, por fim, para o classificador SVM, o qual realizará o reconhecimento.

Fatores externos, como iluminação, impactam diretamente o display em medidores digitais e têm menor efeito nas imagens de dígitos de medidores analógicos. Portanto, optou-se por aplicar o pré-processamento somente para aquele tipo de imagens. Nas seções seguintes serão explicadas as fases do método proposto.

\subsection{Pré-processamento}

No momento da captura, fatores externos, como a iluminação, podem interferir diretamente na qualidade da imagem 
adquirida dos dígitos em medidores digitais, resultando, principalmente, em problemas de contraste.

Dadas essas condições, faz-se necessário aplicar técnicas de pré-processamento a fim de melhorar as imagens, favorecendo uma melhor extração das características. Para o método proposto, foi aplicado o melhoramento por otimização de contraste baseada em fusão variacional (VFGLE).

A otimização de contraste baseada em fusão variacional (VFGLE) foi proposta por Tian and Cohen (2018) para melhorar imagens com iluminação não-uniforme de forma adaptativa. Realiza-se melhoramento global e local, os quais são combinados via otimização de contraste e correção de cores, resultando em imagens com níveis de cores mais balanceados. A Figura 5 ilustra resultados da aplicação das técnicas supracidadas em imagens de dígitos provenientes de medidores digitais.

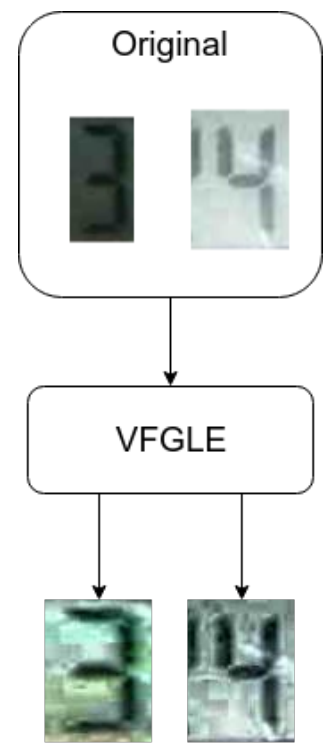

Figura 5. Aplicação da técnica VFGLE para o melhoramento de contraste.

\subsection{Extração de Características}

Para a extração de características, faz-se uso as técnicas Histogram of Oriented Gradients (HoG) e Local SelfSimilarity (LSS).

Características de objetos como forma e aparência locais podem ser bem representadas pelas suas bordas e também pela distribuição das intensidades dos gradientes. O HoG (Dalal and Triggs, 2005) é uma técnica que consegue capturar características locais a despeito de ruídos provenientes da textura, extraindo gradientes e direções de bordas (Yao et al., 2015). Para tornar a extração de características invariante ao tamanho das imagens, o conjunto de células, blocos, ângulos e demais parâmetros do HoG devem ser constantes para cada região extraída (Quintanilha et al., 2017).

O LSS (Shechtman and Irani, 2007) é um descritor local que pode ser utilizado de forma global. Captura a similaridade entre cores, arestas, padrões repetitivos e texturas complexas de uma forma unificada. A imagem é segmentada em vários patches de um dado tamanho. Esses patches são comparados com seus blocos vizinhos em uma área abrangente de determinado tamanho centrada em um ponto $q$. Com isso, é calculada a soma do quadrado das diferenças (SQD) entre os patches. O SQD é normalizado e transformado em uma área de correlação. O resultado é normalizado e projetado em um espaço de intervalos particionados pelo número de intervalo de ângulo e intervalo radial. Por fim, o valor máximo no intervalo de espaço é considerado o valor da característica.

O processo de seleção de características também é abordado neste trabalho a fim de obter um conjunto de atributos menor e mais representativo para as instâncias. Nesta etapa, utiliza-se o algoritmo BestFirst (Hall et al., 2009). Este seletor utiliza uma estratégia gulosa para buscar a melhor combinação de atributos dentro do espaço de características.

\section{RESULTADOS E DISCUSSÃO}

Foram realizados dois experimentos, um utilizando as imagens de dígitos de medidores analógicos e outro com os de medidores digitais. Em cada experimento, foram feitos testes com e sem seleção de características a fim de verificar qual desses produziria os melhores resultados.

A combinação dos descritores HoG e LSS gerou instâncias com 5825 características para ambos os tipos de dígitos. O descritor HoG foi configurado com os seguintes parâmetros: número de blocos igual a 4 , sendo 8 células por bloco com sobreposição igual a 1; e número de direções no histograma igual a 24. Para o LSS, foram utilizados: o tamanho dos patches (5px), o raio para os blocos (40), o número de intervalos radiais dos patches da imagem (3) e o intervalo de ângulos (12). É importante ressaltar que os parâmetros foram definidos empiricamente para ambos os descritores.

As instâncias são submetidas ao classificador SVM (Cortes and Vapnik, 1995) utilizando-se o método de validação cruzada $k$-fold $(k=10)$. Reitera-se que os experimentos foram realizados com cada conjunto de imagens (digital e analógico) separadamente. Logo, foram gerados dois modelos de inferência, um para cada tipo de dígito.

Os experimentos com SVM foram executados com o auxílio da biblioteca Scikit-Learn (Pedregosa et al., 2011) implementada em linguagem Python ${ }^{1}$. Foram realizados testes com e sem seleção de características. Nestes experimentos, a utilização do seletor BestFirst deu-se com o auxílio da ferramente WEKA (Hall et al., 2009).

A Tabela 2 apresenta os resultados obtidos de acurácia para o reconhecimento de dígitos de medidores analógicos. Observa-se que os testes com e sem seleção de características alcançaram acurácia média de 96,30\% e 96,72\%, respectivamente. A etapa de seleção reduziu o conjunto para 200 características.

Observa-se que o teste com seleção de características apresentou o melhor valor de acurácia e também um desvio padrão menor, entre as classes de medidor em comparação com o experimento sem a etapa de seleção. Isso indica um reconhecimento mais equilibrado entre os tipos de

\footnotetext{
1 https://www.python.org/
} 
Tabela 2. Resultados do método proposto para dígitos de medidores analógicos separados por tipo de medidor.

\begin{tabular}{ccc}
\hline Classes & HOG+LSS & $\begin{array}{c}\text { HOG+LSS } \\
\text { com Seleção }\end{array}$ \\
\hline Classe 1 & $91,50 \%$ & $92,50 \%$ \\
\hline Classe 2 & $97,00 \%$ & $96,90 \%$ \\
\hline Classe 3 & $98,20 \%$ & $97,80 \%$ \\
\hline Classe 4 & $96,80 \%$ & $96,50 \%$ \\
\hline Classe 5 & $95,30 \%$ & $97,50 \%$ \\
\hline Classe 6 & $98,10 \%$ & $98,10 \%$ \\
\hline Classe 7 & $97,20 \%$ & $97,80 \%$ \\
\hline Média & $96,30 \% \pm 2,32$ & $\mathbf{9 6 , 7 2 \%} \pm \mathbf{1 , 9 4}$ \\
\hline
\end{tabular}

medidores analógicos não obstante a variabilidade entre as fontes dos dígitos.

Quanto aos experimentos realizados com os dígitos de medidores digitais, foram feitos testes sem melhoramento e com a abordagem VFGLE. Os resultados podem ser vistos na Tabela 3.

Tabela 3. Resultados do método proposto para dígitos de medidores digitais.

\begin{tabular}{ccc}
\hline & HOG+LSS & $\begin{array}{c}\text { HOG+LSS } \\
\text { com Seleção }\end{array}$ \\
\hline $\begin{array}{c}\text { Sem } \\
\text { pré-processamento }\end{array}$ & $97,80 \%$ & $97,10 \%$ \\
\hline VFGLE & $\mathbf{9 7 , 9 0 \%}$ & $\mathbf{9 7 , 6 0 \%}$ \\
\hline
\end{tabular}

O reconhecimento de dígitos de medidores digitais apresentou melhor resultado quando foi aplicada a técnica de VFGLE como melhoramento de imagem, obtendo acurácias de $97,60 \%$ e $97,90 \%$, respectivamente, para os experimentos com e sem seleção de características. A etapa de seleção reduziu o conjunto para 300 caraterísticas. Em contraste aos experimentos com dígitos de medidores analógicos, observa-se na Tabela 3 , que o experimento apresentou melhores resultados com o conjunto original de características.

Ressalta-se, também, que há pouca diferença percentual entre os resultados alcançados pelas abordagens sem préprocessamento e com VFGLE. Baseado nisso, considera-se que optar por não executar a etapa de pré-processamento é algo viável, a fim de diminuir possíveis custos computacionais para a realização da tarefa sem comprometer, abruptamente, o resultado esperado.

Por fim, como citado na Seção 2, este trabalho apresenta-se como uma evolução do trabalho desenvolvido por Quintanilha et al. (2017). A Tabela 4 mostra a comparação dos resultados de acurácia obtidos por esses trabalhos.

Tabela 4. Comparação com trabalhos relacionados.

\begin{tabular}{ccc}
\hline & Med. Analógicos & Med. Digitais \\
\hline Quintanilha et al. (2017) & $79,52 \%$ & - \\
\hline Método Proposto & $\mathbf{9 6 , 7 2 \%}$ & $\mathbf{9 7 . 9 0 \%}$ \\
\hline
\end{tabular}

\section{CONCLUSÃO E TRABALHOS FUTUROS}

Este trabalho apresentou um método para o reconhecimento de dígitos em medidores digitais e analógicos utilizando processamento de imagens e inteligência computacional. O trabalho propôs a utilização combinadas dos descritores HoG e LSS para a extração de características das imagens dos dígitos. O método também utiliza o classificador SVM para a etapa de reconhecimento.

O método apresenta acurácias de $97,90 \%$ e de $96,72 \%$, respectivamente, para o dígitos de medidores digitais e analógicos. Portanto, considerados como resultados bastante promissores para a aplicação ao qual este trabalho será integrado.

Como trabalho futuro, cita-se as tarefas de localização dos displays nos medidores e de segmentação de dígitos. Além da incorporação do método proposto neste trabalho em uma aplicação de autoleitura para dispositivos móveis. Por fim, busca-se investigar também uma solução que utilize de técnicas de aprendizado profundo, como Redes Neurais Convolucionais (CNNs).

\section{REFERÊNCIAS}

ANEEL (2019). Energia no brasil e no mundo. URL http://www2.aneel.gov.br/arquivos/pdf/atlas_ par1_cap2.pdf.

Cortes, C. and Vapnik, V. (1995). Support-vector networks. Machine learning, 20(3), 273-297.

Dalal, N. and Triggs, B. (2005). Histograms of oriented gradients for human detection. In 2005 IEEE Computer Society Conference on Computer Vision and Pattern Recognition (CVPR'05), volume 1, 886-893 vol. 1. doi: 10.1109/CVPR.2005.177.

Hall, M., Frank, E., Holmes, G., Pfahringer, B., Reutemann, P., and Witten, I.H. (2009). The weka data mining software: An update. SIGKDD Explor. Newsl., 11(1), 10-18. doi:10.1145/1656274.1656278. URL http: //doi.acm.org/10.1145/1656274.1656278.

Parthiban, K. and Palanisamy, A. (2013). Reading values in electrical meter using image processing techniques. In Intelligent Interactive Systems and Assistive Technologies (IISAT), 2013 International Conference on, 1-7. IEEE.

Pedregosa, F., Varoquaux, G., Gramfort, A., Michel, V., Thirion, B., Grisel, O., Blondel, M., Prettenhofer, P., Weiss, R., Dubourg, V., Vanderplas, J., Passos, A., Cournapeau, D., Brucher, M., Perrot, M., and Duchesnay, E. (2011). Scikit-learn: Machine learning in Python. Journal of Machine Learning Research, 12, 2825-2830.

Quintanilha, D.B.P., Costa, R.W.S., Diniz, J.O.B., de Almeida, J.D.S., Braz, G., Silva, A.C., de Paiva, A.C., Monteiro, E.M., Froz, B.R., Piheiro, L.P.A., and Melho, W. (2017). Automatic consumption reading on electromechanical meters using hog and svm. In 7th Latin American Conference on Networked and Electronic Media (LACNEM 2017), 57-61. doi:10.1049/ic.2017.0036.

Shechtman, E. and Irani, M. (2007). Matching local self-similarities across images and videos. In 2007 IEEE Conference on Computer Vision and Pattern Recognition, 1-8. doi:10.1109/CVPR.2007.383198.

Tian, Q.C. and Cohen, L.D. (2018). A variationalbased fusion model for non-uniform illumination image enhancement via contrast optimization and color correction. Signal Processing, 153, 210 220. doi:https://doi.org/10.1016/j.sigpro.2018.07. 022. URL http://www.sciencedirect.com/science/ article/pii/S0165168418302548. 
Vidinich, R. and Nery, G. (2009). Pesquisa e desenvolvimento contra o furto de energia. Revista Pesquisa $e$ Desenvolvimento da ANEEL-PED, 15.

Yao, S., Pan, S., Wang, T., Zheng, C., Shen, W., and Chong, Y. (2015). A new pedestrian detection method based on combined hog and lss features. Neurocomputing, 151, 1006 1014. doi:https://doi.org/10.1016/j.neucom.2014.08. 080. URL http://www.sciencedirect.com/science/ article/pii/S0925231214013393.

Zhang, Y., Yang, S., Su, X., Shi, E., and Zhang, H. (2016). Automatic reading of domestic electric meter: an intelligent device based on image processing and zigbee/ethernet communication. Journal of Real-Time Image Processing, 12(1), 133-143. 